\title{
BOUNDARY BEHAVIOR OF $\mathrm{BMO}\left(B_{n}\right)$
}

BY

\author{
PAULA A. RUSSO ${ }^{1}$
}

\begin{abstract}
If $f$ is a holomorphic function of bounded mean oscillation in the unit ball of $\mathbf{C}^{\prime \prime}$, then it has radial limits at almost every point of the boundary of the ball. The question remains as to how nicely one can expect this function to behave on subsets of the boundary of zero measure. For example, there is a holomorphic BMO function in the ball that has a finite radial limit at no point of the $n$-torus. We show here that this is not an isolated phenomenon; there exists at least one other $n$-dimensional submanifold of the boundary of the ball with this same behavior.
\end{abstract}

Let $B_{n}=\left\{z \in \mathbf{C}^{n}:|z|<1\right\}$ be the unit ball of $\mathbf{C}^{n}$, and let $\sigma_{n}$ denote the normalized Lebesgue measure on $\partial B_{n}$. We are interested in the following question: Given a submanifold $M$ of $\partial B_{n}$ and a function $f$ holomorphic in $B_{n}$, what conditions on $f$ will guarantee that $f$ has radial limits almost everywhere on $M$ ? It is a classical result, see [6], for example, that if $f$ is in the Hardy space $H^{p}\left(B_{n}\right)$, for $0<p \leqslant \infty$, then $f$ has radial limits almost everywhere on $\partial B_{n}$. If $f \in H^{\infty}\left(B_{n}\right)$, then more can be said. For example, Nagel and Rudin proved in [4] that if $\varphi:[a, b] \rightarrow B_{n}$ is a nowhere complex tangential curve and $\varphi^{\prime}$ satisfies a Lipschitz condition, then $f$ has radial limits almost everywhere on $\varphi$ with respect to arclength measure. It was recently shown by Ahern [1] that there exists a holomorphic function of bounded mean oscillation with radial limits at no point of the $n$-torus $T_{n}=\left\{z \in \partial B_{n}\right.$ : $\left.\left|z_{j}\right|=1 / \sqrt{n}\right\}$. Motivated by this, we show the existence of another $n$-dimensional submanifold of $\partial B_{n}$ with this same behavior.

We define the holomorphic functions of bounded mean oscillation as follows. If $f \in H^{2}\left(B_{n}\right)$ we say that $f \in \operatorname{BMO}\left(B_{n}\right)$ if there exists a constant $C$ such that for all $F \in H^{2}\left(B_{n}\right)$

$$
\left|\int_{\partial B_{n}} F \tilde{f} d \sigma_{n}\right| \leqslant C\|F\|_{1} .
$$

It follows that $H^{\infty}\left(B_{n}\right) \subset \mathrm{BMO}\left(B_{n}\right) \subset H^{p}\left(B_{n}\right)$ for $0<p<\infty$. (For more information on $\operatorname{BMO}\left(B_{n}\right)$ for $n>1$, see [2].)

Let $\pi$ be the mapping from $\mathbf{C}^{n}$ into $\mathbf{C}$ given by $\pi\left(z_{1}, z_{2}, \ldots, z_{n}\right)=\sum_{j=1}^{n} z_{j}^{2}$. One can easily verify that $\pi\left(B_{n}\right)=B_{1}$ and $\pi\left(\bar{B}_{n}\right)=\bar{B}_{1}$. We set $W_{n}=\pi^{-1}\left(\partial B_{1}\right) . W_{n}$ is then a submanifold of $\partial B_{n}$ with real dimension $n$, and it is this submanifold which we will consider. Note that for $n=2, W_{n}$ is biholomorphically equivalent to $T_{n}$ via the mapping $\left(z_{1}, z_{2}\right) \rightarrow\left(\left(z_{1}-i z_{2}\right) / \sqrt{2},\left(z_{1}+i z_{2}\right) / \sqrt{2}\right)$.

Received by the editors March 28, 1985.

1980 Mathematics Subject Classification. Primary 32E35; Secondary 32A40.

${ }^{1}$ This work represents a part of the author's doctoral dissertation completed under the guidance of Herbert Alexander. 
To construct the desired function, we show that if $g$ is holomorphic in $B_{1}$ with the property that $\sup _{|\lambda|<1}\left|f^{\prime}(\lambda)\right|(1-|\lambda|)<\infty$, then $g \circ \pi \in \operatorname{BMO}\left(B_{n}\right)$. (A function $g$ with this property is called Bloch function.) It is known that there exists such a function $G$ with radial limits at no point of $\partial B_{1}$ [5]. It follows that $G \circ \pi$ will have a radial limit at no point of $\pi^{-1}\left(\partial B_{1}\right)=W_{n}$. It thus remains to prove

THEOREM 1. If $g$ is a Bloch function, then $g \circ \pi \in \operatorname{BMO}\left(B_{n}\right)$.

We begin with a calculation which will be the main tool in the proof of Theorem 1 .

THEOREM 2. For each integer $n \geqslant 2$, there exists a constant $K_{n}$ such that if $f$ is $a$ continuous complex-valued function on $B_{1}$, then

$$
\int_{\partial B_{n}} f \circ \pi d \sigma_{n}=K_{n} \int_{0}^{2 \pi} \int_{0}^{1} f\left(r e^{i \theta}\right) r\left(1-r^{2}\right)^{(n-3) / 2} d r d \theta
$$

We evaluate the left-hand side of (1) by applying the following reformulation of the co-area formula of Federer [3].

Proposition. If $M$ and $N$ are real Riemannian submanifolds, with $\operatorname{dim}_{\mathbf{R}} M=m \geqslant$ $n=\operatorname{dim}_{\mathbf{R}} N$, and $f$ is a smooth map, then

$$
\int_{M} g(x) d \mu(x)=\int_{N}\left[\int_{f^{-1}\{y\}} \frac{g(x)}{J_{n} f(x)} d H^{m-n}(x)\right] d H^{n}(y),
$$

where $\mu$ is Lebesgue measure on $M, H^{k}$ is $k$-dimensional Hausdorff measure, and $g$ is a Lebesgue integrable function on $M$ satisfying the conditions

(i)

$$
\int_{N}\left[\int_{f^{-1}\{y\}}\left|\frac{g(x)}{J_{n} f(x)}\right| d H^{m-n}(x)\right] d H^{n}(y)<\infty
$$

and

(ii) there exists $E_{j} \subset M$ such that $E_{1} \subset E_{2} \subset \cdots, \mu\left(\cup_{j=1}^{\infty} E_{j}\right)=1$, and $\left(g / J_{n} f\right) \chi_{E,} \in L^{1}(d \mu)$ for each $j$.

Applying (2) to $M=S^{2 n-1}$ and $N=B_{1}$ we obtain

$$
\int_{S^{2 n-1}} f \circ \pi d \sigma_{n}=C_{n} \int_{B_{1}} f(\lambda) \int_{\Gamma_{\lambda}} \frac{1}{J_{2} \pi(x)} d H^{2 n-3}(x) d \lambda,
$$

where the constant $C_{n}$ depends only on $n, \Gamma_{\lambda}=\pi^{-1}\{\lambda\} \cap S^{2 n-1}$, and $f$ is continuous on $B_{1}$. In polar coordinates this becomes

$$
\int_{S^{2 n-1}} f \circ \pi d \sigma_{n}=C_{n} \int_{0}^{2 \pi} \int_{0}^{1} r f\left(r e^{i \theta}\right)\left[\int_{\Gamma_{r e^{\prime \theta}}} \frac{1}{J_{2} \pi(x)} d H^{2 n-3}(x)\right] d r d \theta .
$$

Thus, our proof of Theorem 2 will be complete if we show that

$$
\int_{\Gamma_{r e^{\prime} \theta}} \frac{1}{J_{2} \pi(x)} d H^{2 n-3}(x)=C_{n}\left(1-r^{2}\right)^{(n-3) / 2} .
$$

This will be accomplished in the following lemmas. 
LeMma 1. $J_{2} \pi(\xi)=4 \sqrt{1-|\pi(\xi)|^{2}}$.

Proof. Since $J_{2} \pi=\frac{1}{2}\|d \pi \wedge d \bar{\pi}\|$, we must show that $\|d \pi(\xi) \wedge d \bar{\pi}(\xi)\|=$ $8 \sqrt{1-|\pi(\xi)|^{2}}$.

Let $\left\{t_{j}\right\}_{j=1}^{2 n-1}$ be an orthonormal basis of $T S^{2 n-1}(\xi)$, the real tangent space of $S^{2 n-1}$ at the point $\xi$. Let $\left\{w_{j}\right\}_{j=1}^{2 n-1}$ be the corresponding dual basis of the cotangent space. Then we have $d \pi=\sum_{j=1}^{2 n-1}\left(t_{j} \pi\right) w_{j}$. Hence, to prove the lemma, we construct such a basis and compute $d \pi$.

We choose our tangent vectors to be of the form

$$
\begin{array}{ll}
t_{2 l}(\xi)=\operatorname{Re}\left(\sum_{k=1}^{n} \alpha_{k}^{l}(\xi) \frac{\partial}{\partial \xi_{k}}\right), & 1 \leqslant l \leqslant n-1, \\
t_{2 l+1}(\xi)=\operatorname{Im}\left(\sum_{k=1}^{n} \alpha_{k}^{l}(\xi) \frac{\partial}{\partial \xi_{k}}\right), & 0 \leqslant l \leqslant n-1
\end{array}
$$

for $\alpha^{l} \in \mathbf{C}^{n}$. One can easily check that the $t_{j}$ 's will be orthonormal if and only if

$$
\left|\alpha^{l}(\xi)\right|=2 \text { and }\left\langle\alpha^{l}(\xi), \alpha^{m}(\xi)\right\rangle=0 .
$$

Furthermore, in order for each $t_{j}(\xi)$ to be an element of $T S^{2 n-1}(\xi)$, we must have $t_{j}\left(\sum_{k=1}^{n}\left|\xi_{k}\right|^{2}\right)=0$. This implies that

$$
\left\langle\alpha^{\prime}(\xi), \xi\right\rangle=0 \quad \text { for } 1 \leqslant l \leqslant n-1 \quad \text { and } \quad \operatorname{Im}\left\langle\alpha^{0}(\xi), \xi\right\rangle=0 .
$$

Let $\xi \in S^{2 n-1}$ so that $|\pi(\xi)|<1$. Let $\alpha^{0}(\xi)=2 \xi$ and $\alpha^{1}(\xi)=2 q /|q|$, where $q=\bar{\xi}-\langle\bar{\xi}, \xi\rangle \xi$ is the component of $\bar{\xi}$ orthogonal to $\xi$. By (5) and (6) we see that $\left\{t_{1}(\xi), t_{2}(\xi), t_{3}(\xi)\right\}$ is an orthonormal set of tangent vectors for each $\xi \in S^{2 n-1}$, with $|\pi(\xi)|<1$.

We now choose $\alpha^{3}(\xi), \ldots, \alpha^{n-1}(\xi) \in \mathbf{C}^{n}$ so that (5) and (6) are satisfied. This will imply that $\alpha^{l}(\xi)$ is orthogonal to both $\alpha^{1}(\xi)$ and $\xi$ for $2 \leqslant l \leqslant n-1$, and hence we have

$$
\left\langle\alpha^{\prime}(\xi), \bar{\xi}\right\rangle=0, \quad 2 \leqslant l \leqslant n-1 .
$$

To compute $d \pi$ we have

$$
\begin{aligned}
d \pi & =\sum_{j=1}^{2 n-1}\left(t_{j} \pi\right) w_{j} \\
& =\left(t_{1} \pi\right) w_{1}+\sum_{l=1}^{n-1}\left(\left(t_{2 l} \pi\right) w_{2 l}+\left(t_{2 l+1} \pi\right) w_{2 l+1}\right) \\
& =-i\left\langle\alpha^{0}, \bar{\xi}\right\rangle w_{1}+\sum_{l=1}^{n-1}\left(\left\langle\alpha^{l}, \bar{\xi}\right\rangle w_{2 l}-i\left\langle\alpha^{l}, \bar{\xi}\right\rangle w_{2 l+1}\right),
\end{aligned}
$$

which by (7) gives

$$
d \pi=-i\left\langle\alpha^{0}, \bar{\xi}\right\rangle w_{1}+\left\langle\alpha^{1}, \bar{\xi}\right\rangle w_{2}-i\left\langle\alpha^{1}, \bar{\xi}\right\rangle w_{3} .
$$

Hence,

$$
\begin{aligned}
d \pi \wedge d \bar{\pi}=-2 i( & \operatorname{Re}\left[\left\langle\alpha^{0}, \bar{\xi}\right\rangle\left\langle\overline{\alpha^{1}, \bar{\xi}}\right\rangle\right] w_{1} \wedge w_{2} \\
& \left.-\operatorname{Im}\left[\left\langle\alpha^{0}, \bar{\xi}\right\rangle\left\langle\overline{\alpha^{1}, \bar{\xi}}\right\rangle\right] w_{1} \wedge w_{3}-\left|\left\langle\alpha^{1}, \bar{\xi}\right\rangle\right|^{2} w_{2} \wedge w_{3}\right)
\end{aligned}
$$


and

$$
\|d \pi \wedge d \bar{\pi}\|^{2}=4\left|\left\langle\alpha^{1}, \bar{\xi}\right\rangle\right|^{2}\left(\left|\left\langle\alpha^{0}, \bar{\xi}\right\rangle\right|^{2}+\left|\left\langle\alpha^{1}, \bar{\xi}\right\rangle\right|^{2}\right)
$$

This gives

$$
\begin{aligned}
\|d \pi \wedge d \bar{\pi}\| & =2\left(2 \sqrt{1-|\pi(\xi)|^{2}}\right)\left(4|\pi(\xi)|^{2}+4\left(1-|\pi(\xi)|^{2}\right)\right)^{1 / 2} \\
& =8 \sqrt{1-|\pi(\xi)|^{2}} .
\end{aligned}
$$

Thus

$$
J_{2} \pi(\xi)=4 \sqrt{1-|\pi(\xi)|^{2}} \quad \text { for all } \xi \in S^{2 n-1} \backslash W_{n}
$$

and hence for all $\xi \in S^{2 n-1}$.

LEMMA 2.

$$
\int_{\Gamma_{\lambda}} d H^{2 n-3}=C_{n}\left(1-|\lambda|^{2}\right)^{(n-2) / 2} \text { for } 0 \leqslant|\lambda|<1
$$

PRoof. Since $\Gamma_{r}=e^{i \theta / 2}\left(\Gamma_{r e^{\prime \theta}}\right)$, and the volume of $\Gamma_{r}$, represented by the left-hand integral, is rotation invariant, it is sufficient to prove the lemma for $\lambda=r$.

Suppose $z=X+i Y \in \Gamma_{r}$ where $X, Y \in \mathbf{R}^{n}$. It follows that $z \in \Gamma_{r}$ if and only if

$$
|X|^{2}=\frac{1+r}{2}, \quad|Y|^{2}=\frac{1-r}{2} \text { and }\langle X, Y\rangle=0 \text {. }
$$

To evaluate $\int_{\Gamma_{r}} d H^{2 n-3}$ we again use the co-area formula. We consider the map $f$ : $\Gamma_{r} \rightarrow \sqrt{(1+r) / 2} S^{n-1}$ defined by $f(X+i Y)=X$. By the above comments we know that $f$ maps $\Gamma_{r}$ onto $\sqrt{(1+r) / 2} S^{n-1}$. Thus, applying (2) for $g \equiv 1$, we have

(9) $\int_{\Gamma_{r}} d H^{2 n-3}=\int_{\sqrt{(1+r) / 2} S^{n} 1}\left[\int_{f^{-1}\{p\}} \frac{1}{J_{n-1} f(x)} d H^{n-2}(x)\right] d H^{n-1}(p)$.

To compute $J_{n-1} f$, we construct an orthonormal basis of $T \Gamma_{r}(z)$ at $z=X+i Y$.

For $1 \leqslant l \leqslant n-2$ let $\alpha^{l} \in \mathbf{R}^{n}$ such that the set $\left\{\sqrt{2 /(1-r)} Y, \alpha^{1}, \ldots, \alpha^{n-2}\right\}$ is an orthonormal basis of $T S^{n-1}(X)$. Note that this is possible since $\langle X, Y\rangle=0$. Let

$$
\begin{aligned}
t_{1} & =\operatorname{Im}\left[\frac{2}{\sqrt{1-r^{2}}} \sum_{j=1}^{n}\left(z_{j}-r \bar{z}_{j}\right) \frac{\partial}{\partial z_{j}}\right], \\
t_{2 l} & =\operatorname{Re}\left[\sum_{j=1}^{n} 2 \alpha_{j}^{l} \frac{\partial}{\partial z_{j}}\right], \quad t_{2 l+1}=\operatorname{Im}\left[\sum_{j=1}^{n} 2 \alpha_{j}^{l} \frac{\partial}{\partial z_{j}}\right] .
\end{aligned}
$$

Because of our choice of the $\alpha^{l}$ 's, it follows that the $t_{k}$ 's are orthonormal. To see that they are tangent vectors, we must show that

$$
t_{k}\left(\sum_{j=1}^{n} z_{j}^{2}\right)=t_{k}\left(\sum_{j=1}^{n} z_{j} \bar{z}_{j}\right)=0
$$


Since these are the defining functions of $\Gamma_{r}$. We have

$$
\begin{aligned}
t_{1}\left(\sum_{j=1}^{n} z_{j}^{2}\right) & =\frac{-i}{\sqrt{1-r^{2}}} \sum_{j=1}^{n}\left(z_{j}-r \bar{z}_{j}\right) 2 z_{j} \\
& =\frac{-i}{\sqrt{1-r^{2}}}\left(2 \pi(z)-2 r|z|^{2}\right)=0, \\
t_{1}\left(\sum_{j=1}^{n} z_{j} \bar{z}_{j}\right) & =\frac{-i}{\sqrt{1-r^{2}}} \sum_{j=1}^{n}\left(z_{j}-r \bar{z}_{j}\right) \bar{z}_{j}-\left(\bar{z}_{j}-r z_{j}\right) z_{j} \\
& =\frac{-i r}{\sqrt{1-r^{2}}}(\overline{-\pi(z)}+\pi(z))=0, \\
t_{2 l}\left(\sum_{j=1}^{n} z_{j}^{2}\right) & =\frac{1}{2} \sum_{j=1}^{n}\left(2 \alpha_{j}^{l}\right)\left(2 z_{j}\right)=2\left\langle\alpha^{l}, \bar{z}\right\rangle=0, \\
t_{2 l}\left(\sum_{j=1}^{n} z_{j} \bar{z}_{j}\right) & =\sum_{j=1}^{n} \alpha_{j}^{l} \bar{z}_{j}+\alpha_{j}^{l} z_{j}=2\left\langle\alpha^{l}, X\right\rangle=0
\end{aligned}
$$

and similarly for $t_{2 l+1}$. Thus the $t_{k}$ 's do form an orthonormal basis of $T \Gamma_{r}(z)$.

We now evaluate $t_{k} f_{j}$ where $f=\left(f_{1}, f_{2}, \ldots, f_{n}\right)$. We have

$$
t_{1} f_{j}=\sqrt{\frac{1+r}{1-r}} y_{j} ; \quad t_{2 l} f_{j}=\alpha_{j}^{l} ; \quad t_{2 l+1} f_{j}=0 \text {. }
$$

Thus, $\Lambda^{n-1} d f$ is zero on each basis vector of $\Lambda_{n-1}\left(T \Gamma_{r}\right)$ with the exception of $t_{1} \wedge t_{2} \wedge t_{4} \wedge \cdots \wedge t_{2 n-4}$. Let $S_{j}$ denote the permutation group on $\{1,2, \ldots, j-1$, $j+1, \ldots, n\}$. If $\tau \in S_{j}$, let $\tau_{1}, \ldots, \tau_{n-1}$ denote the values $\tau(1), \tau(2), \ldots$, $\tau(j-1), \tau(j+1), \ldots, \tau(n)$, respectively. We have

$$
\begin{array}{rl}
\Lambda^{n-1} & d f\left(t_{1} \wedge t_{2} \wedge t_{4} \wedge \cdots \wedge t_{2 n-4}\right) \\
& =\sum_{j=1}^{n} \sum_{\tau \in S_{j}}(-1)^{|\tau|}\left(t_{1} f_{\tau_{1}}\right) \cdots\left(t_{2 n-4} f_{\tau_{n-1}}\right) \\
& =\sum_{j=1}^{n} \sum_{\tau \in S_{j}}(-1)^{|\tau|}\left(\sqrt{\frac{1+r}{1-r}}\right) y_{\tau_{1}} \alpha_{\tau_{2}}^{1} \cdots \alpha_{\tau_{n-1}}^{n-2}
\end{array}
$$

which is equal to $\sqrt{(1+r) /(1-r)}\left(Y \wedge \alpha^{1} \wedge \cdots \wedge \alpha^{n-2}\right)$. Hence,

$$
\begin{aligned}
J_{n-1} f & =\left\|\Lambda^{n-1} d f\right\|=\frac{1+r}{1-r}\left\|Y \wedge \alpha^{1} \wedge \cdots \wedge \alpha^{n-2}\right\| \\
& =\frac{\sqrt{1+r}}{2},
\end{aligned}
$$

since $\left\{\sqrt{2 /(1-r)} Y, \alpha^{1}, \ldots, \alpha^{n-2}\right\}$ is an orthonormal family. Thus (9) becomes

$$
\int_{\Gamma_{r}} d H^{2 n-3}=\int_{\sqrt{(1+r) / 2}} S^{n-1}\left[\int_{f^{-1}\{p\}} \sqrt{\frac{2}{1+r}} d H^{n-2}(x)\right] d H^{n-1}(p) .
$$


Since $f^{-1}\{p\}=\{(p, Y):|Y|=\overline{(1-r) / 2},\langle p, Y\rangle=0\}$ is an $(n-2)$-sphere we have

$$
\int_{f{ }^{1}\{p\}} d H^{n-2}=C_{n}\left(\frac{1-r}{2}\right)^{(n-2) / 2} .
$$

Hence

$$
\begin{aligned}
\int_{\Gamma_{r}} d H^{2 n-3} & =C_{n}\left(\frac{1+r}{2}\right)^{(n-1) / 2}(1+r)^{-1 / 2}\left(\frac{1-r}{2}\right)^{(n-2) / 2} \\
& =C_{n}\left(1-r^{2}\right)^{(n-2) / 2} .
\end{aligned}
$$

This proves Lemma 2, which together with Lemma 1 proves Theorem 2.

We now proceed to the proof of Theorem 1. It is sufficient to show that if $g$ is a Bloch function on the unit disk, then there exists a constant $A=A(g)$ such that for all holomorphic polynomials we have

$$
\left|\int_{\partial B_{n}} F(\overline{g \circ \pi}) d \sigma_{n}\right| \leqslant A\|F\|_{1} .
$$

The method used to prove (10) follows closely that used by Ahern in [1].

Let $T g=g \circ \pi$ and let $d \mu_{n}=K_{n} r\left(1-r^{2}\right)^{(n-2) / 2} d r d \theta$, where $K_{n}$ is the constant guaranteed by Theorem 3. It follows from this theorem that if $g$ is continuous on $B_{1}$, then

$$
\int_{\partial B_{n}}|T g|^{p} d \sigma_{n}=\int_{B_{1}}|g|^{p} d \mu_{n} .
$$

Thus $T$ extends uniquely to be an isometry of $L^{p}\left(d \mu_{n}\right)$ into $L^{p}\left(d \sigma_{n}\right)$ for all finite $p$; in particular, for $p=2$. This implies that if $F$ is a holomorphic polynomial, then

$$
\int_{\partial B_{n}} F(\overline{T g}) d \sigma_{n}=\int_{B_{1}}\left(T^{*} F\right) \bar{g} d \mu_{n}
$$

where $T^{*}$ is the Hilbert space adjoint of the isometry from $L^{2}\left(d \mu_{n}\right)$ into $L^{2}\left(d \sigma_{n}\right)$. So, to prove (10), it is sufficient to prove

$$
\left|\int_{B_{1}}\left(T^{*} F\right) \bar{g} d \mu_{n}\right| \leqslant A\|F\|_{1}
$$

for all holomorphic polynomials $F$. This will be done in two steps. The first is to show that if $F$ is a holomorphic polynomial in $\mathrm{C}^{n}$, then $T^{*} F$ is a holomorphic polynomial in $\mathrm{C}$ and $\int_{B_{1}}\left|T^{*} F\right| d \mu_{n} \leqslant \int_{\partial B_{n}}|F| d \sigma_{n}$. The second step is to show that if $g$ is a Bloch function then there exists a constant $A=A(g)$ such that for any holomorphic polynomial $h$ of one variable we have

$$
\left|\int_{B_{1}} h \bar{g} d \mu_{n}\right| \leqslant A \int_{B_{1}}|h| d \mu_{n} .
$$

From these two steps the theorem clearly follows. 
LEMMA 3. If $F(z)$ is a holomorphic polynomial, then so is $T^{*} F(\lambda)$. Moreover, $\int_{B_{1}}\left|T^{*} F\right| d \mu_{n} \leqslant \int_{\partial B_{n}}|F| d \sigma_{n}$.

Proof. To prove the first part of the lemma, it is sufficient to consider only the holomorphic monomials $z^{\alpha}$. We begin by making three observations:

(i) If $\alpha=\left(\alpha_{1}, \alpha_{2}, \ldots, \alpha_{n}\right)$ is any multi-index and $\tau(\alpha)$ is any permutation of the $\alpha_{j}$ 's, then $T^{*}\left(z^{\alpha}\right)=T^{*}\left(z^{\tau(\alpha)}\right)$. This follows from the fact that $\tau$ induces a unitary transformation on $\partial B_{n}$ which leaves both $\pi$ and $\sigma_{n}$ invariant and so we have

$$
\int_{\partial B_{n}} z^{\alpha}(\overline{g \circ \pi}) d \sigma_{n}=\int_{\partial B_{n}} z^{\tau(\alpha)}(\overline{g \circ \pi}) d \sigma_{n},
$$

from which the assertion follows.

(ii) $T^{*}\left(z^{\alpha}\right)=0$ if $\alpha_{j}$ is odd for any $j$. Because both $\sigma_{n}$ and $\pi$ are left invariant when $z_{j}$ is replaced by $-z_{j}$ we have

$$
\int_{\partial B_{n}} z^{\alpha}(\overline{g \circ \pi}) d \sigma_{n}=\int_{\partial B_{n}}(-1)^{\alpha_{j}} z^{\alpha}(\overline{g \circ \pi}) d \sigma_{n}
$$

and thus $T^{*}\left(z^{\alpha}\right)=0$.

(iii)

$$
T^{*}\left(z_{1}^{2 K} z_{2}^{2 L}\left(z^{\prime}\right)^{2 \beta}\right)=\frac{2 K-1}{2 L+1} T^{*}\left(z_{1}^{2 K-2} z_{1}^{2 L+2}\left(z^{\prime}\right)^{2 \beta}\right)
$$

for all nonnegative integers $K$ and $L ; K>0$, and all multi-indices $\beta$ of the form $\beta=\left(\beta_{1}, \ldots, \beta_{n-2}\right)$. (Note that $z^{\prime}=\left(z_{3}, z_{4}, \ldots, z_{n}\right)$.)

We consider the unitary transformation

$$
\left(z_{1}, z_{2}, \ldots, z_{n}\right) \rightarrow\left(z_{1} \cos \theta+z_{2} \sin \theta, z_{1} \sin \theta-z_{2} \cos \theta, z_{3}, \ldots, z_{n}\right)
$$

for $\theta \in \mathbf{R}$. It is easily verified that $\pi$ is invariant under this transformation, as is $\sigma_{n}$. Hence, we have

$$
\begin{aligned}
& \int_{\partial B_{n}} z_{1}^{s} z_{2}^{t}\left(z^{\prime}\right)^{2 \beta}(\overline{g \circ \pi}) d \sigma_{n} \\
& \quad=\int_{\partial B_{n}}\left(z_{1} \cos \theta+z_{2} \sin \theta\right)^{s}\left(z_{1} \sin \theta-z_{2} \cos \theta\right)^{t}\left(z^{\prime}\right)^{2 \beta}(\overline{g \circ \pi}) d \sigma_{n}
\end{aligned}
$$

for all nonnegative integers $s$ and $t$ and for all real $\theta$. Differentiating both sides of (12) with respect to $\theta$ gives

$$
\begin{aligned}
0=\int_{\partial B_{n}}(g \circ \pi)\left(z^{\prime}\right)^{2 \beta}\left[-s\left(z_{1} \cos \theta+z_{2} \sin \theta\right)^{s-1}\left(z_{1} \sin \theta-z_{2} \cos \theta\right)^{t+1}\right. \\
\\
\left.\quad+t\left(z_{1} \cos \theta+z_{2} \sin \theta\right)^{s+1}\left(z_{1} \sin \theta-z_{2} \cos \theta\right)^{t-1}\right] d \sigma_{n} .
\end{aligned}
$$

By (12) this implies that

$$
\int_{\partial B_{n}} s z_{1}^{s-1} z_{2}^{t+1}\left(z^{\prime}\right)^{2 \beta}(\overline{g \circ \pi}) d \sigma_{n}=\int_{\partial B_{n}} t z_{1}^{s+1} z_{2}^{t-1}\left(z^{\prime}\right)^{2 \beta}(\overline{g \circ \pi}) d \sigma_{n} .
$$

Thus,

$$
s T^{*}\left(z_{1}^{s-1} z_{2}^{t+1}\left(z^{\prime}\right)^{2 \beta}\right)=t T^{*}\left(z_{1}^{s+1} z_{2}^{t-1}\left(z^{\prime}\right)^{2 \beta}\right),
$$

and choosing $s=2 K-1$ and $t=2 L+1$, we obtain our assertion. 
For an arbitrary monomial $z^{\alpha}$, it follows from (i) and successive applications of (iii) that

$$
T^{*}\left(z^{2 \alpha}\right)=C_{\alpha, \beta} T^{*}\left(z^{2 \beta}\right) \text { for all } \beta \text { such that }|\beta|=|\alpha| \text {. }
$$

By Theorem 3 we know that

$$
\int_{\partial B_{n}}[\pi(z)]^{K}(\overline{g \circ \pi}) d \sigma_{n}=\int_{B_{1}} \lambda^{K} \bar{g} d \mu_{n},
$$

and thus $T^{*}\left([\pi(z)]^{K}\right)=\lambda^{K}$. Expanding $[\pi(z)]^{K}$ gives

$$
\lambda^{K}=T^{*}\left(\left(z_{1}^{2}+\cdots+z_{n}^{2}\right)^{K}\right)=\sum_{|\alpha|=K} \frac{K !}{\alpha !} T^{*}\left(z^{2 \alpha}\right) ;
$$

hence by (13) we have

$$
T^{*}\left(z^{2 \alpha}\right)=C_{\alpha} \lambda^{|\alpha|}
$$

for some constant $C_{\alpha}$. This completes the proof of the first part of the lemma.

Since $T: L^{p}\left(d \mu_{n}\right) \rightarrow L^{p}\left(d \sigma_{n}\right), 1 \leqslant p<\infty$, is an isometry, it follows that $T^{*}$ : $L^{q}\left(d \sigma_{n}\right) \rightarrow L^{q}\left(d \mu_{n}\right), 1<q \leqslant \infty$, has norm at most 1 . Thus, if $F$ is a holomorphic polynomial in $n$ variables it follows that

$$
\int_{B_{1}}\left|T^{*} F\right|^{q} d \mu_{n} \leqslant \int_{\partial B_{n}}|F|^{q} d \sigma_{n}
$$

for each $q>1$. Letting $q \rightarrow 1$, we have, by the dominated convergence theorem,

$$
\begin{aligned}
\int_{B_{1}}\left|T^{*} F\right| d \mu_{n} & =\lim _{q \rightarrow 1^{+}} \int_{B_{1}}\left|T^{*} F\right|^{q} d \mu_{n} \\
& \leqslant \lim _{q \rightarrow 1^{+}} \int_{\partial B_{n}}|F|^{q} d \sigma_{n}=\int_{\partial B_{n}}|F| d \sigma_{n} .
\end{aligned}
$$

Lemma 4. Let $g$ be a Bloch function in the unit disk. Then there exists a constant $A=A(g)$ such that, for any holomorphic polynomial $h$ of one variable,

$$
\left|\int_{B_{1}} h \bar{g} d \mu_{n}\right| \leqslant A \int_{B_{1}}|h| d \mu_{n} .
$$

This is just Lemma 2.3 in [1] with $w_{n}(r)=K_{n} r\left(1-r^{2}\right)^{(n-3) / 2}$. This completes the final step of the proof of Theorem 2 .

\section{REFERENCES}

1. P. Ahern, On the behavior near a torus of functions holomorphic in the ball, Pacific J. Math. 107 (1983), $267-278$.

2. R. R. Coifman, R. Rochberg and G. Weiss, Factorization theorems for Hardy spaces in several iariables, Ann. of Math. (2) 103 (1976), 611-635.

3. H. Federer, Curvature measures, Trans. Amer. Math. Soc. 93 (1959), 418-491.

4. A. Nagel and W. Rudin, Local boundary behavior of bounded holomorphic functions, Canad. J. Math. 30 (1978), 583-592.

5. C. Pommerenke, On Bloch functions, J. London Math. Soc. 2 (1970), 689-695.

6. W. Rudin, Function theory in the unit ball of $\mathbf{C}^{n}$, Springer-Verlag, New York and Berlin, 1980.

Department of Mathematics, Michigan State University, East Lansing, Michigan 48824

Current address: Department of Mathematics, C138 Padelford Hall, GN-50, University of Washington, Seattle, Washington 98195 